\title{
25. THÉORIE DES ANOMALIES D'EFFET TUNNEL \\ DUE A DES IMPURETÉS A L'INTÉRIEUR D'ÉLECTRODES MÉTALLIQUES
}

\author{
F. MEZEI et A. ZAWADOWSKI
}

Central Research Institute for Physics, Budapest, Hongrie

L'effet d'une couche d'impuretés atomiques placées à l'intérieur de l'une des électrodes d'une jonction tunnel est étudié par la méthode des fonctions de Green. La diffusion résonnante des électrons de conduction crée un pic en résistance à une polarisation correspondant à l'énergie de résonance. On considère que la couche d'impuretés se trouve à l'intérieur de l'électrode à une distance de l'ordre de $\xi_{\Delta}$ de la surface de jonction où $\xi_{\Delta}$ est déterminé par la variation du moment avec l'amplitude de diffusion. 